\title{
DNA-lipid systems. A physical chemistry study
}

R. Dias ${ }^{1}$, F. Antunes ${ }^{1}$, M. Miguel ${ }^{1}$, S. Lindman ${ }^{2}$ and B. Lindman²
1Departamento de Q uímica, U niversidade de Coimbra, Coimbra, Portugal 2Physical Chemistry 1, Center for Chemistry and Chemical Engineering, Lund University, Lund, Sweden

\section{Correspondence \\ B. Lindman \\ Physical Chemistry 1 \\ Center for Chemistry and \\ Chemical Engineering \\ Lund University \\ P.O. Box 124 \\ 22100 Lund \\ Sweden \\ E-mail: mgmiguel@ci.uc.pt or \\ bjorn.lindman@ fkem1.lu.se \\ Presented at the XXX Annual Meeting of the Brazilian Society of Biochemistry and Molecular Biology, Caxambu, MG, Brazil, May 19-22, 2001.}

Research supported by JNICT and Praxis XXI (PRAXIS/BD/21227/99),

Fundação para a Ciência e Tecnologia (FCT, Project Sapiens PCTI/99/Q UI/35414), Portugal,

Swedish Research Council for

Engineering Sciences (TFR), and Center for Amphiphilic Polymers (CAP), Lund, Sweden.

Received November 5, 2001 Accepted February 26, 2002

\section{Abstract}

It is well known that the interaction of polyelectrolytes with oppositely charged surfactants leads to an associative phase separation; however, the phase behavior of DNA and oppositely charged surfactants is more strongly associative than observed in other systems. A precipitate is formed with very low amounts of surfactant and DNA. DNA compaction is a general phenomenon in the presence of multivalent ions and positively charged surfaces; because of the high charge density there are strong attractive ion correlation effects. Techniques like phase diagram determinations, fluorescence microscopy, and ellipsometry were used to study these systems. The interaction between DNA and catanionic mixtures (i.e., mixtures of cationic and anionic surfactants) was also investigated. We observed that DNA compacts and adsorbs onto the surface of positively charged vesicles, and that the addition of an anionic surfactant can release DNA back into solution from a compact globular complex between DNA and the cationic surfactant. Finally, DNA interactions with polycations, chitosans with different chain lengths, were studied by fluorescence microscopy, in vivo transfection assays and cryogenic transmission electron microscopy. The general conclusion is that a chitosan effective in promoting compaction is also efficient in transfection.

\section{Introduction}

The interactions in mixed solutions of DNA with surfactants or polyelectrolytes as co-solutes have been attracting a rapidly increasing interest among physicists, chemists and biologists as candidates for vectors of gene transfection (Figure 1) (1), but the systems are also of general physicochemical relevance.

Most of our work is related to the interactions between DNA and quaternary ammo- nium surfactants. Our main motivation in these studies is the use of our knowledge of the physical chemistry of amphiphiles in single and complex surfactant systems and polymer-surfactants in general, to understand the efficiency of the DNA compaction they induce (DNA is highly compacted within the cells). We are interested in the complexes formed and also in the use of negative surfactants to reverse the compaction progress. It should be noted that these amphiphiles in general behave like the lipids used for gene 
delivery, so they can be used as models that are less expensive and easier to work with.

In the present report, we review our findings about DNA-surfactant systems and the understanding we have obtained after recently starting to work in the area.

\section{DNA displays a strong associative behavior with cationic surfactants}

Phase diagrams are significant not only in telling us about conditions of stability of different phases but also in reporting on intermolecular/interaggregate interactions. The different aspects of phase separation phenomena in polymer-surfactant systems have only recently become understood and investigated; they are found to be closely analogous to the more widely studied systems of two polymers in a solvent.

For the case of interest here, that of an oppositely charged polyelectrolyte-surfactant pair, there is in general an associative phase separation over wide concentration regions, i.e., there is a separation into one phase rich in both polymer and surfactant and one, often highly dilute solution phase. This phase separation is entropically driven, determined by the translational entropy of the counterions. At higher surfactant concentrations there is generally miscibility, i.e., "redissolution" takes place.

The surfactant molecules are in an aggregated form in the concentrated phase, but different types of aggregates are formed in different systems; micelles of different shape, cubic liquid crystalline phase, (normal or inverse) hexagonal phase and lamellar phase are the cases described so far (2-9). The factors determining the type of aggregate formed can be assumed to be the same as for simple surfactant systems, a statement supported by some recent reports (8).

If the electrostatic driving force for association is eliminated or screened, a segregative phase separation, characteristic of sys-
Figure 1. A hypothetical model of gene transfection. Three possible methods of cell entry are indicated. Reprinted from Ref. 1, with permission.

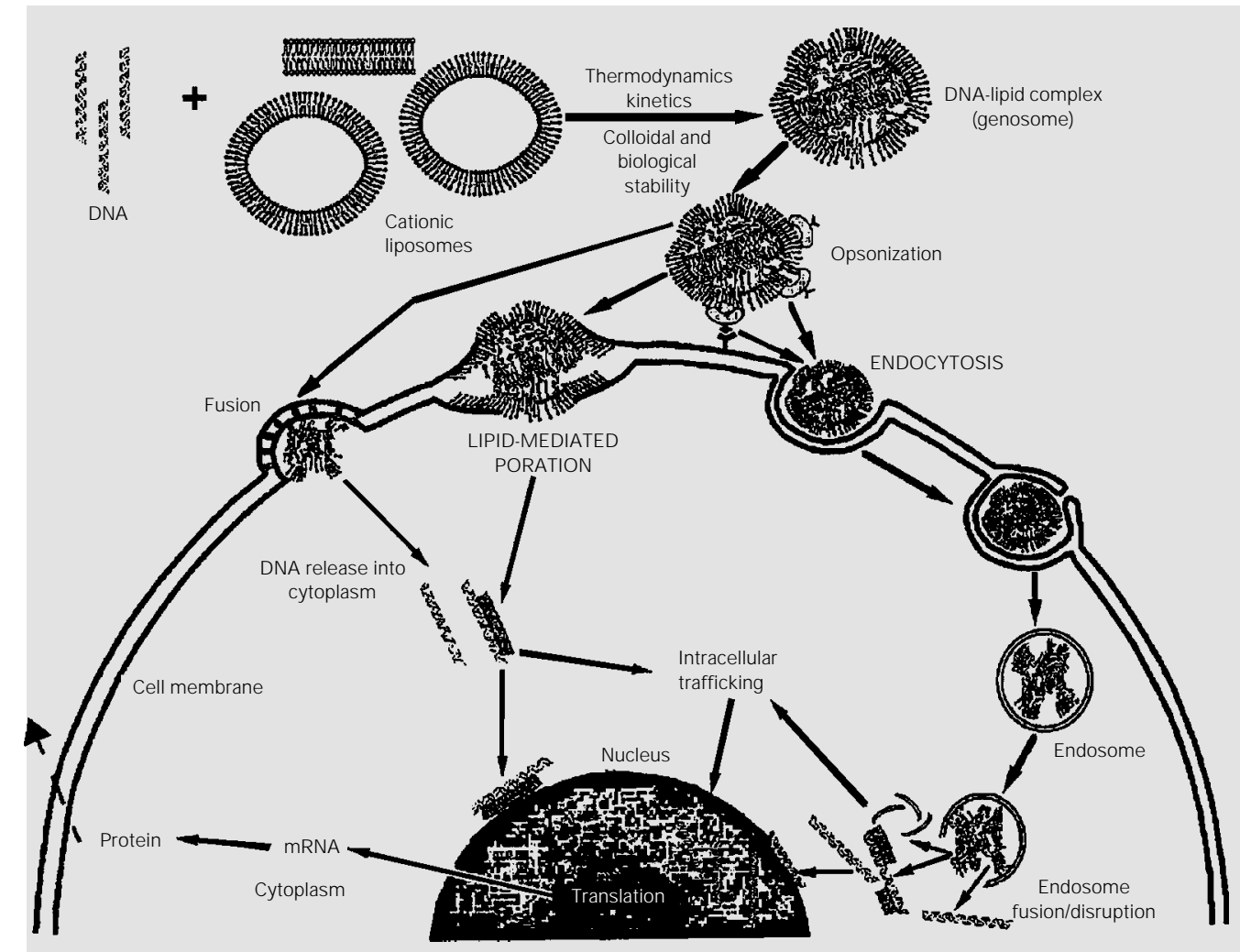


tems with a low entropic driving force of mixing, can be expected (9). Indeed it has been observed for certain polyion-ionic surfactant systems that two solution phases are formed, one enriched in polymer and the other in surfactant, when large amounts of electrolyte have been added. At intermediate salt concentrations, there is no phase separation (10).

DNA-cationic surfactant systems only partly appear to display this behavior (11). As exemplified in the phase diagrams in Figures 2 and 3, there is a strong associative behavior. The associative phase separation is enhanced as the alkyl chain length of the surfactant increases. This has also been observed for other polymer-surfactant systems and is in line with a lower critical micellar concentration/critical aggregation concentration $(\mathrm{CMC} / \mathrm{CAC})$ and a larger surfactant aggregate for longer alkyl chains $(12,13)$. We also observed, as previously reported (14), that a short surfactant like octyltrimethylammonium bromide (OTAB), although able to increase the turbidity of the system (Figure 3) and hence to form complexes, does not precipitate to a large extent. In the case of a shorter chain there is no visible interaction between the surfactant and DNA.

Nevertheless, some features are different compared to previous studies of polyelectrolyte-surfactant interactions: 1) associative phase separation starts at very low concentrations of DNA or surfactant, 2) there is no redissolution at high surfactant concentrations, and 3) there is no redissolution on addition of salt; on the contrary, depending on salt concentration, phase separation can increase.

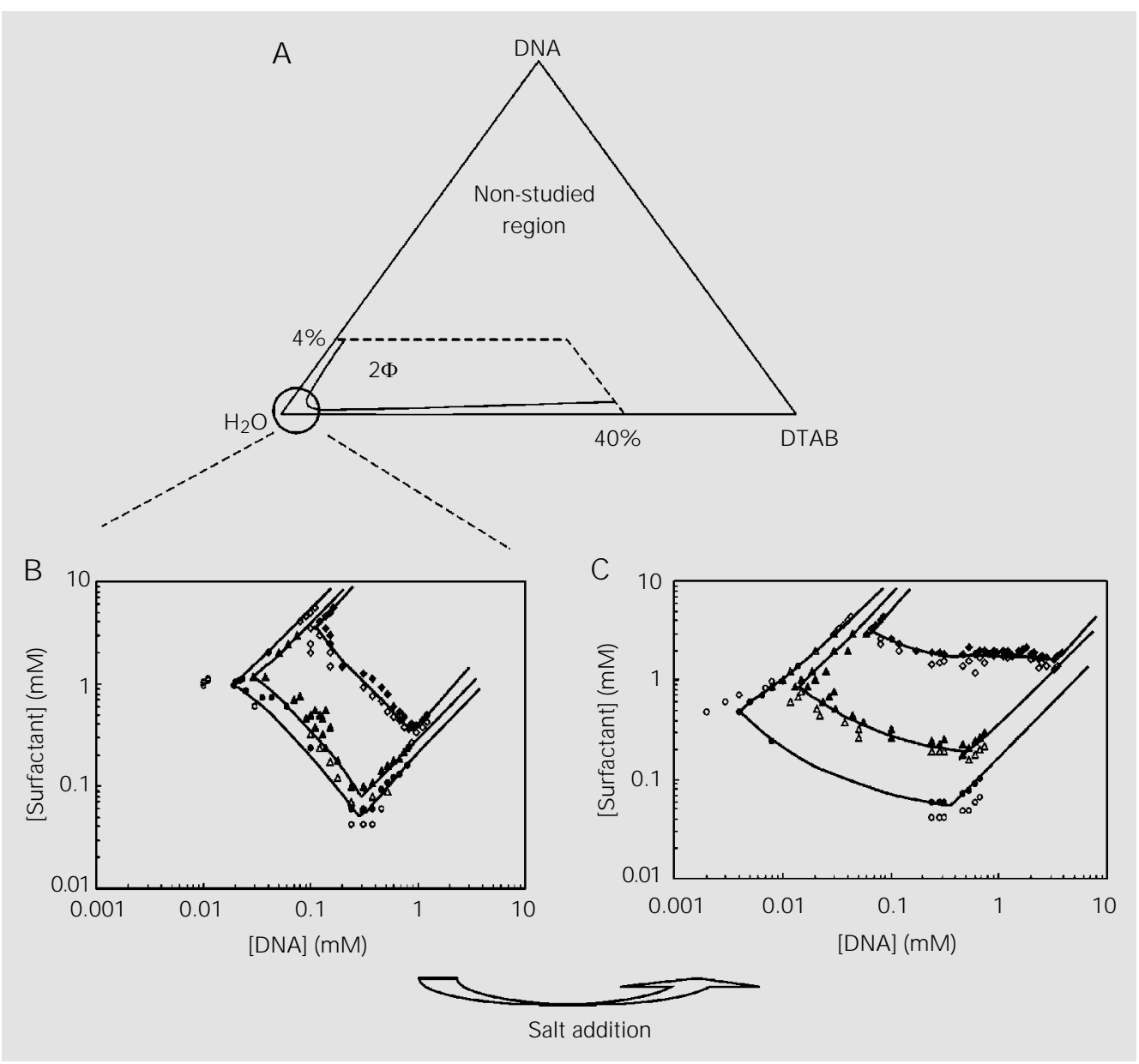

Figure 2. A, Schematic representation of the isothermal pseudoternary phase diagram for the DNA-dodecyltrimethylammonium bromide (DTAB)-water system. There is a phase separation into two phases in almost the entire region considered. B, Expanded view of the water corner of the system (diamond) including the DNA-tetradecyltrimethylammonium bromide (TTAB)-water (triangle) and DNAcetyltrimethylammonium bromide (CTAB)-water (circle) systems for comparison. Open symbols refer to the clear onephase solutions and filled symbols to two-phase samples. C, Phase map for the same three systems in the presence of 0.1 $\mathrm{M} \mathrm{NaBr}$. $\mathrm{T}=25^{\circ} \mathrm{C}$. Redrawn from Ref. 11, with permission. 
Surfactant binding to polymers is in general cooperative as shown by the fact that the bound surfactant occurs in an aggregated form, being often found or assumed to be in the form of discrete spherical micelles. Observations for other polyion systems imply that the surfactant aggregates are more or less evenly distributed over different polymer molecules. However, for DNA the observations seem to imply a different behavior, i.e., a very uneven distribution of surfactant between different DNA molecules (1517). In turn, such a "double cooperativity" implies that surfactant binding to a DNA molecule facilitates further binding. The mechanism for this is not clear but should be analyzed in terms of extended ("infinite") self-assemblies formed and of the polyion compaction due to the attractive electrostatic correlation effects discussed below. Such compaction concentrates the polymer ionic groups in space, possibly leading to a preference for further surfactant binding relative to the "naked" DNA molecules.

The other two observations mentioned need to be further studied; the observed "insolubility" may correspond to a quantitative rather than qualitative difference from other polymers and might then be possibly rationalized in terms of the very high linear charge density $(18,19)$, one charge per $1.7 \AA$ (for the double-helix B-form) (14).

The salt effect was shown to be complex (11) since the associative phase separation could be both enhanced and decreased depending on the conditions. Interestingly, further studies have shown that we have reached the point where physical chemistry and biology "collide". As physical chemists we try to keep the system as simple as possible. Our three-component system, or better, pseudoternary component system, which is DNA/ surfactant/water, is already sufficiently complicated to study (see below). But some precautions are necessary when working with biological molecules like DNA. In this particular case some electrolyte is necessary to keep the molecule in its native state (doublehelix). Without such additives the DNA strands drift apart due to electrostatic repul-
Figure 3. Same as Figure 2B with results on the DNA decyltrimethylammonium bromide (DeTAB)-water (squares) and DNA-octyltrimethylammonium bromide (OTAB)-water (crosses) systems. The open symbols correspond to one-phase solutions and the filled symbols to twophase samples. $\mathrm{T}=25^{\circ} \mathrm{C}$.

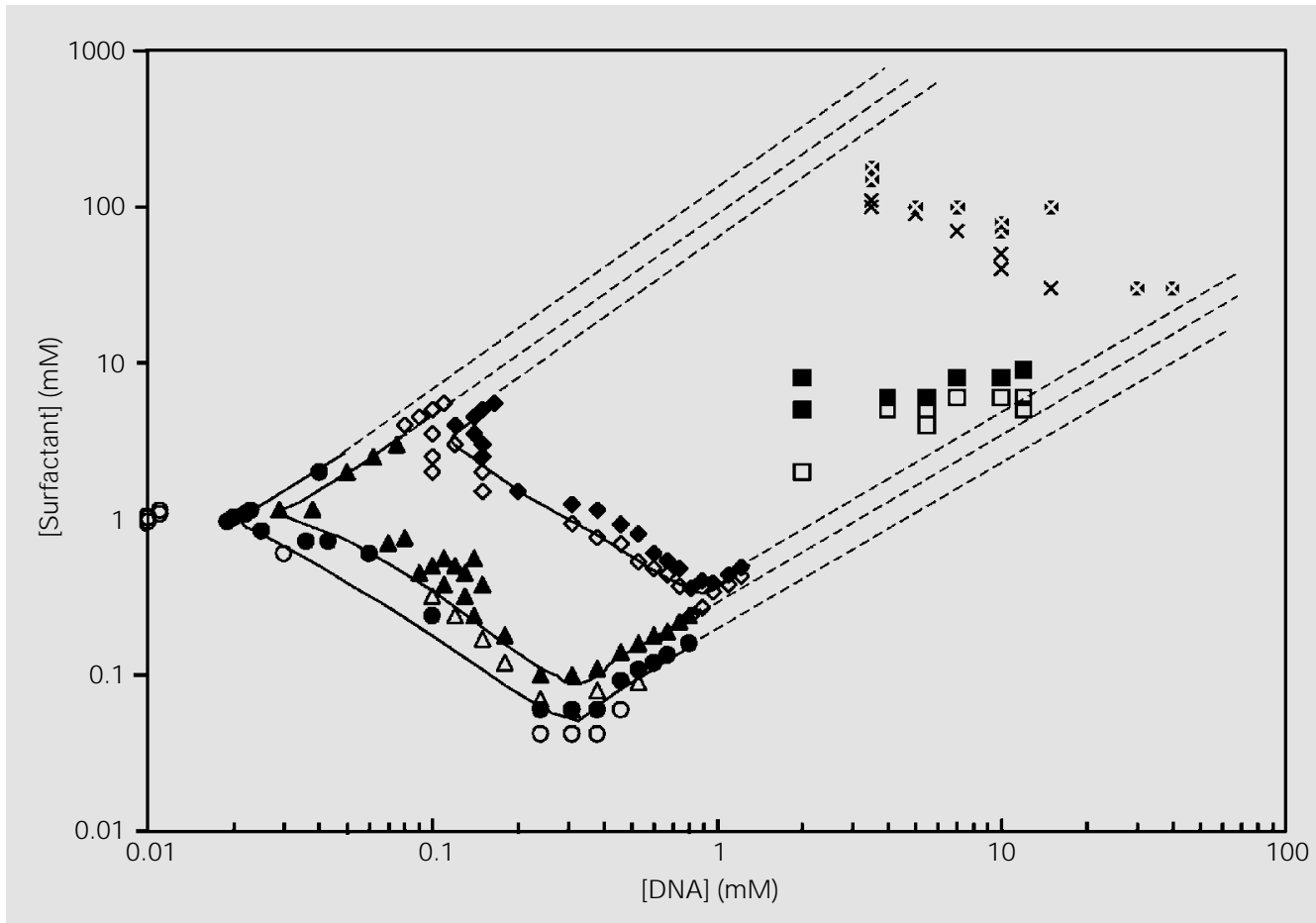


sions (14). This could indeed explain the unexpected results obtained: we have different phase behaviors because we do have DNA in two different conformations, doublehelix and denatured single stranded. Further studies on phase diagrams are being conducted in our laboratory taking into consideration the salt and temperature effects on DNA denaturation.

Let us finally point out that our description of phase diagrams in two-dimensional plots is basically incorrect and can be misleading. A system of two electrolytes, without common counterions, and a solvent in general cannot be treated as a ternary system. One consequence is that the compositions of separate phases do not usually lie on the "conventional" mixing plane used (cf. Figure 2). The preferred presentation is in a pyramidal phase diagram $(20,21)$, as illustrated in Figure 4. Unfortunately, even a sketchy three-dimensional phase diagram determination is a considerable undertaking.

\section{Attractive ion-ion correlation effects for DNA in the presence of multivalent ions}

The interaction between DNA and oppositely charged surfactants or polyelectrolytes is dominated by electrostatic interactions. For charged systems in general - surfactant self-assemblies, polyelectrolyte, surfaces the Poisson-Boltzmann approach has offered a standard and widely accepted treatment which has provided a broad understanding of the interactions (22); it has very successfully described different aspects of polyionsurfactant interactions.

However, as shown early by $\mathrm{H}$. Wennerström and Bo Jönsson and their coworkers (23), the Poisson-Boltzmann treatment becomes inappropriate for highly charged systems in the presence of multivalent counterions. Here the assumption of uncorrelated positions of the counterions does not hold any longer. The role of counterion correla- tions as charge density and counterion valency increase is best illustrated by an example from recent calculations by Khan et al. $(24,25)$, an example with direct relevance for DNA. Figure 5 shows the end-to-end distance for a polymer as it is progressively charged up. With a monovalent counterion we have the familiar electrostatic expansion leading to an increased end-to-end distance. However, with multivalent counterions there is not only an inhibition of the expansion but the polymer chain becomes more compact as it is charged than when it is uncharged: there is a compaction of the polyion due to attractive counterion correlation effects. These attractive electrostatic correlation effects have been well documented for widely different systems, including ionic surfactant systems with divalent or more highly charged counterions $(26,27)$.

These effects are central in our studies of DNA-surfactant-polyion systems and of
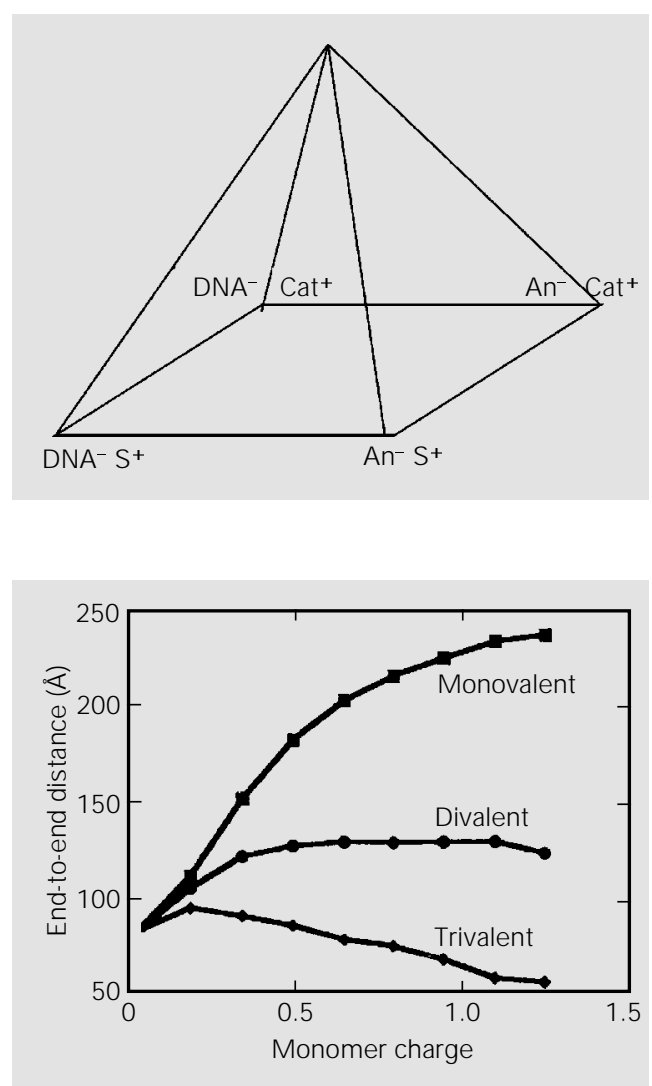

Figure 4. Pyramid representation of the phase diagram for a system of solvent (water), DNA salt and surfactant. Four monovalent ions are present: $\mathrm{DNA}^{-}$(DNA polyanion), $\mathrm{S}^{+}$(surfactant cation), $\mathrm{An}^{-}$(simple anion), and $\mathrm{Cat}^{+}$ (simple cation).

Figure 5. The end-to-end distance is shown as a function of monomer charge for different valences of the counterions. No salt was added $\left(\mathrm{N}=80, \mathrm{C}_{\mathrm{m}}=5.4\right.$ $\mathrm{mM}$, and $\mathrm{b}=6 \AA$ ). Reprinted from Ref. 25, with permission. 
Figure 6. Fluorescence images of T4 DNA molecules moving freely in the buffer solution with the stepwise addition of cetyltrimethylammonium bromide (CTAB). Existence of coil conformation of T4 DNA molecules (A), globule conformation (B), and coexistence of coil state and globule state (C). Reprinted from Ref. 30, with permission.
DNA compaction in general. Since surfactant molecules are self-assembled in their interaction with DNA they will effectively appear as multivalent counterions.

\section{DNA is compacted by cationic surfactants}

The compaction of DNA can be conveniently monitored by fluorescence microscopy, a technique established some time ago $(28,29)$ and which has become widely employed. The
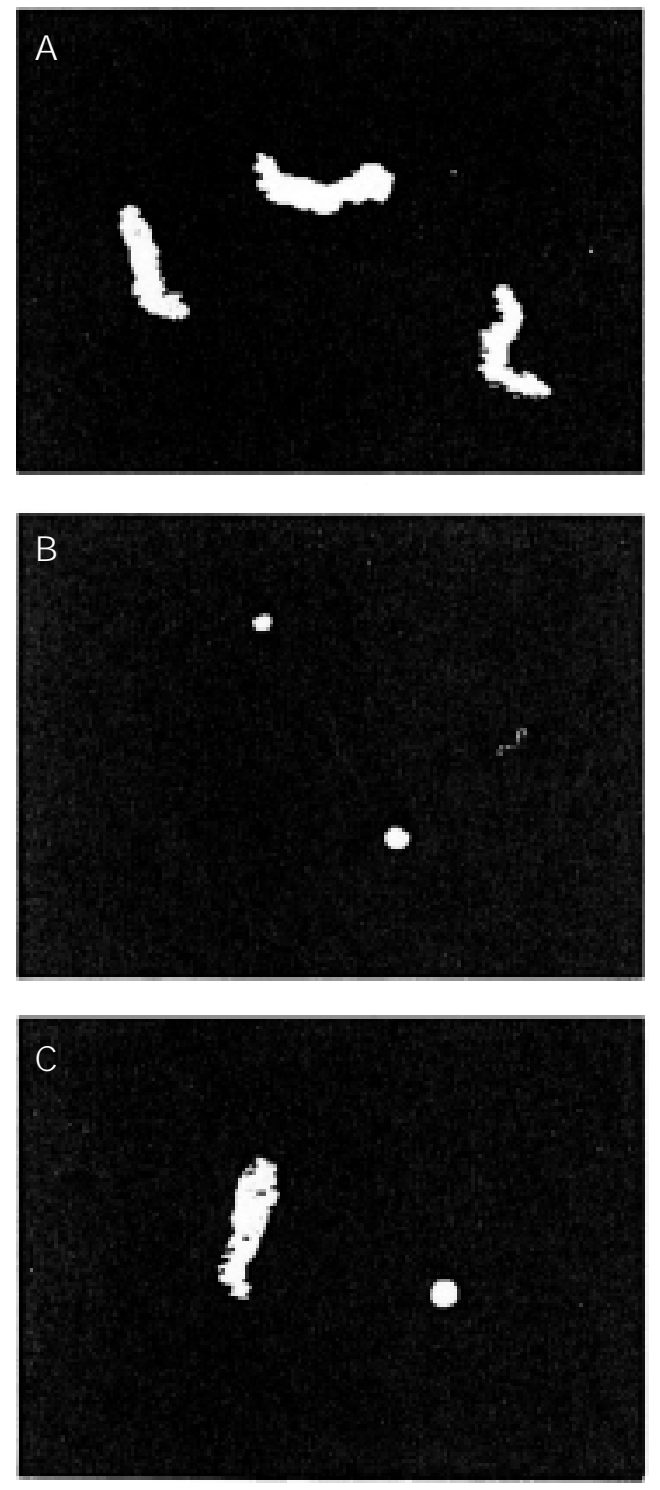

$10.0 \mu \mathrm{m}$ general aspects of DNA compaction by cationic surfactants have been monitored by Yoshikawa's group $(6,15,16,30-32)$ and others and by our group recently $(11,33)$. Some illustrative fluorescence micrographs are presented in Figure 6. The important observations are: 1) on addition of a cationic surfactant to a DNA solution there is a compaction of DNA molecules; 2) compaction is typically not gradual for individual DNA molecules but occurs in a narrow range of surfactant concentrations; 3) at high surfactant concentrations, there is a compaction of all DNA molecules. In a broad intermediate concentration range there is coexistence of extended DNA molecules, "coils", and compacted ones, "globules"; 4) compaction is correlated with the binding isotherm; 5) as the surfactant alkyl chain length increases, compaction occurs at a lower surfactant concentration; 6) compaction can be reversed upon addition of electrolytes.

It seems that, in view of the high molecular weight of DNA, we can to some extent regard this compaction as an associative phase separation at the single molecule level. We see indeed that this "microscopic phase behavior" parallels the macroscopic one reviewed above, with the possible exception of item 6 , which deserves further attention.

The most notable observation appears to be the coexistence of coils and globules in a sizable concentration range. This leads to the same observation as made in conjunction with the phase diagrams: surfactant molecules are very unevenly distributed between DNA molecules and binding shows a cooperativity above that of surfactant self-assembly, i.e., the onset of surfactant binding to one DNA molecule facilitates further binding. This can again be discussed in terms of the attractive electrostatic interactions and the large size of surfactant aggregates formed. However, the latter effect is apparently not required since in studies of DNA compaction induced by other co-solutes, organic solvents, electrolytes, etc., a coexistence of coils and globules is also found $(25,34)$. 


\section{DNA compaction can occur on the surface of cationic vesicles}

Mixed systems of DNA and different types of vesicles have been rather widely investigated (1). An important approach of these studies has been the potential use of such systems as transfection vectors and the bilayers of the vesicles modeling the bilayers of cell membranes. Vesicles of different charges have been investigated, with a focus on cationic ones.

Essentially all of these studies have concerned vesicles which are not thermodynamically stable. In our group, we have been investigating for some time vesicles in mixed cationic + anionic surfactant systems which form spontaneously, have an apparently unlimited life-time and are believed to be thermodynamically stable $(21,35)$. The vesicles are polydisperse with sizes in the approximate range of 0.05 to $0.5 \mu \mathrm{m}$ although there are also a few larger ones; the larger ones can be visualized directly by light microscopy while the smaller ones have been conveniently studied by cryogenic transmission electron microscopy $(36,37)$. In our work we have introduced investigations of the interactions between these "catanionic" vesicles and DNA (38).

In the phase diagram (39) there are two regions of stable vesicles; in one they have a net negative charge and in the other a net positive charge. In solutions of anionic vesicles and DNA, no interaction is observed; for example, there is no compaction of DNA. This indicates that there is no extraction of cationic surfactant from the mixed vesicles, at least for low DNA concentrations, and suggests that the surfactant-surfactant interaction is stronger than the surfactant-DNA interaction.

If DNA is mixed with cationic vesicles, on the other hand, a strong interaction is clearly demonstrated. A direct demonstration is the compaction of DNA. For the larger vesicles, both the compacted DNA and the vesicles can be visualized under a light microscope (Figure 7). Interestingly, DNA compaction occurs in a wide range of conditions on the surface of intact vesicles. By monitoring the temporal replacement of DNA in fluorescence microscopy studies, it was easy to distinguish from DNA compacted onto vesicles or DNA compaction involving micelles. The self-diffusion is slower by orders of magnitude in the case of vesicles. There are two nontrivial points in this respect. First, why is DNA compacted into a globular state and does not associate and extend over the vesicle surface? Second, why does the vesicle state remain intact?

Under conditions of higher DNA concentrations, the situation becomes different, including vesicle disruption, vesicle-vesicle flocculation, and the formation of a precipitate with a lamellar structure. Similar effects have been reported for other vesicle systems (7).

\section{DNA-surfactant aggregates display a rich interfacial behavior}

The interfacial behavior of mixed cat-
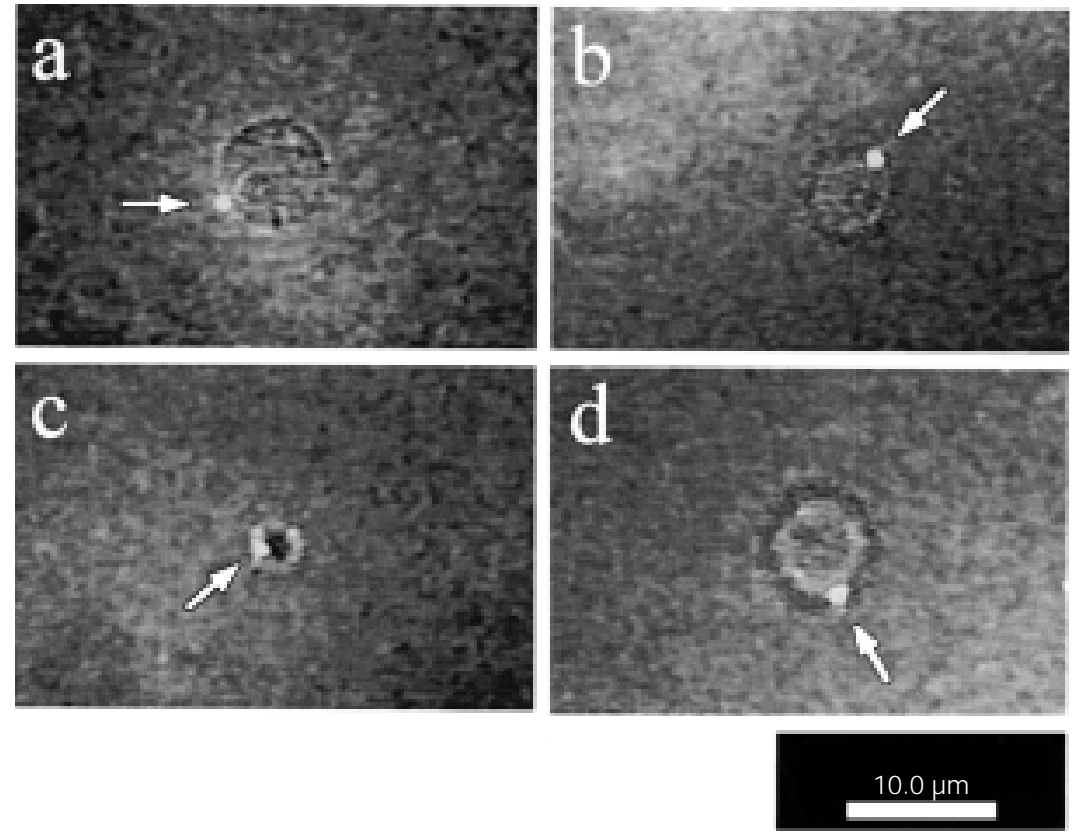

Figure 7. Light microscopy images of single T4 DNA globules adsorbed onto the surface of positively charged catanionic vesicles. Arrows indicate single DNA globules. Reprinted from Ref. 38, with permission. 
ionic surfactant-DNA systems clearly depends strongly on the surface. DNA adsorbs strongly to cationic surfaces and weakly to nonpolar surfaces but shows no adsorption to negatively charged surfaces. Cationic surfactants adsorb strongly to anionic and nonpolar surfaces, but show no adsorption to positively charged surfaces.

In situ null ellipsometry investigations have been started (40) in an attempt to elucidate basic aspects of the adsorption as well as of the structure of the adsorbed layer in mixed systems. By this technique, both the (total) adsorbed amount and the thickness of the adsorbed layer can be monitored in the presence of solutions. Both equilibrium/ steady-state adsorption and kinetics of adsorption and desorption were investigated. Two types of surfaces were used, an anionic polar surface, silica, and a nonpolar hydrophobic surface, hydrophobized silica, both in combination with DNA of different molecular weights and with hexadecyltrimethylammonium bromide (CTAB) as surfactant.

On the polar surfaces there is an adsorption of aggregates between low molecular

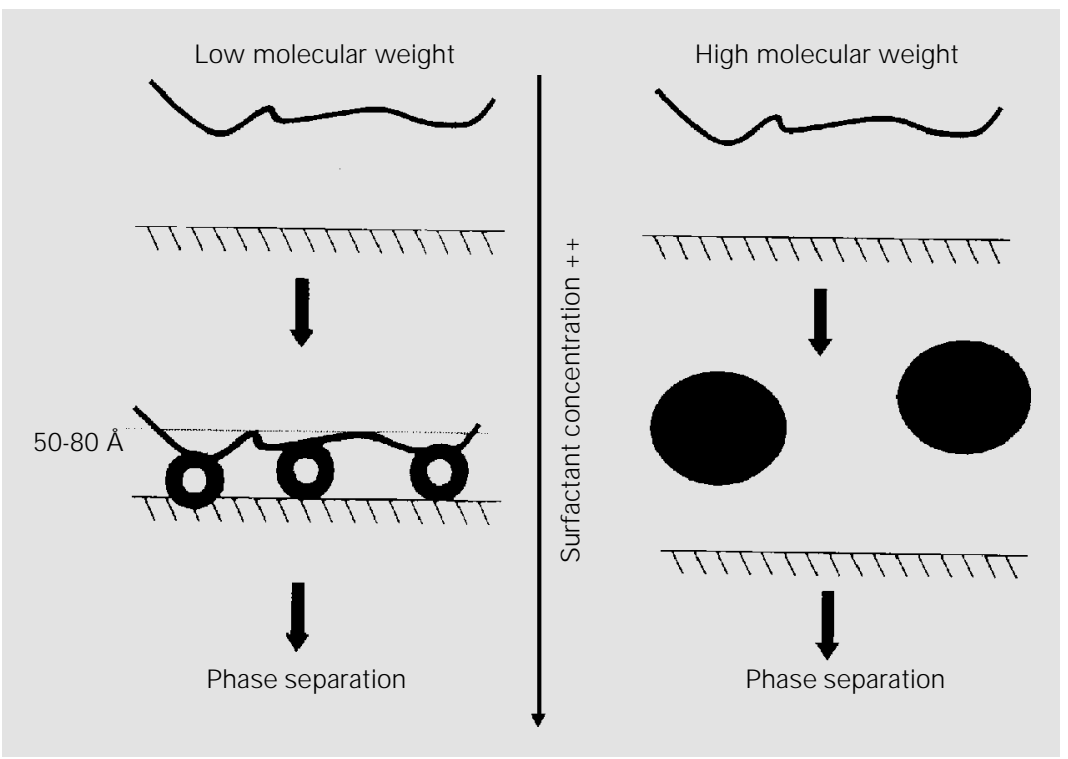

Figure 8. Schematic representation of DNA-cetyltrimethylammonium bromide (CTAB) adsorption on silica. For low molecular weight DNA, adsorption is observed for a wide range of $C T A B$ concentrations. With high molecular weight DNA, precipitation appears to dominate over a very wide range of CTAB concentrations. weight DNA and CTAB while no adsorption is detected with high molecular weight DNA. CTAB adsorbs both in the absence and presence of DNA but with low molecular weight DNA adsorption is facilitated, as shown by a displacement of the adsorption isotherm to considerably lower concentrations. With low molecular weight DNA, further addition of CTAB leads to precipitation; however, with high molecular weight DNA precipitation appears to dominate over adsorption over a very wide range of $\mathrm{CTAB}$ concentrations; this is in line with the "double cooperativity" of surfactant binding inferred from the phase diagram and compaction studies discussed above. The adsorption characteristics are schematically displayed in Figure 8.

Intriguing findings were obtained for hydrophobic surfaces. Both co-solutes adsorb individually, with noncooperative characteristics and when DNA is preadsorbed, addition of CTAB leads to surfactant adsorption to the polymer layer. Interestingly, measurements of the thickness of the surfactant layer show dramatic changes in the structure of the adsorbed layer. This is demonstrated by the opposite variations of adsorbed amount and layer thickness illustrated in Figure 9. When a solution of low molecular weight DNA contacts the surface there is a slow adsorption into a very open layer characterized by a plateau adsorption of ca. $0.2 \mathrm{mg} / \mathrm{m}^{2}$ and a thickness of ca. $20 \mathrm{~nm}$. As surfactant is added, the adsorbed amount greatly increases to almost $3 \mathrm{mg} / \mathrm{m}^{2}$ but the adsorbed layer compacts very strongly. The effect is reversed by rinsing with water: with rinsing, surfactant desorption is fast but polymer desorption is slower by orders of magnitude. Therefore, the adsorbed layer becomes depleted with respect to surfactant and reverts progressively to a highly expanded layer of DNA alone.

These studies clearly demonstrate the possibility of monitoring central aspects of DNA conformational behavior and its compaction on surfaces and demonstrate clear 
analogies between the bulk and the interface behavior.

\section{DNA compaction can be triggered by pH for certain surfactants}

The clearest and best understood example of DNA delivery and DNA compaction is represented by cationic surfactants. In attempts to control delivery, the most suitable appears to be to control the charge of the surfactant (aggregate). This can be achieved most naturally in mixed surfactant systems by varying the mixing ratio or by employing a surfactant with a degree of protonation which varies with $\mathrm{pH}$ in a relevant range. We consider here a surfactant, dodecyldimethylamine oxide (DDAO), which is cationic at low $\mathrm{pH}$ and nonionic at high $\mathrm{pH}$. The $\mathrm{pK}_{\mathrm{a}}$ value of the monomeric surfactant is 5.0 $(41,42)$ but will depend on the state of aggregation.

DNA compaction was studied by fluorescence microscopy as a function of $\mathrm{pH}$ for the surfactant in micellar form (Figure 10) and in vesicular form (in mixture with the bilayer-forming lipid dioleoylphosphatidylethanolamine) (33). At low $\mathrm{pH}$, where the surfactant is cationic, compaction is easily induced at low concentrations of surfactant, while at high $\mathrm{pH}$ even high concentrations of surfactant are unable to induce compaction. In a broad $\mathrm{pH}$ range, the concentration needed for compaction increases strongly with $\mathrm{pH}$ and there is a coexistence of the coil and globular forms of DNA over wide concentration ranges.

A significant observation is that vesicles are much more efficient than micelles in inducing compaction; lower concentrations are needed and the change over from globules to coils occurs at a higher $\mathrm{pH}$ for vesicles. There are different possible causes for this such as the higher aggregation number of the vesicles, leading to a higher total charge number even if the cationic surfactant is diluted by the zwitterionic one. However, probably more important is the fact that, in the case of vesicles, the surfactant aggregates are preexisting, while in the case of micelles, surfactant self-assembly is induced by the polyion, with a concomitant free energy cost.

\section{The structure of DNA-surfactant complexes is important}

Due to hydrophobic interactions, surfactants, like other amphiphiles, self-assemble in aqueous systems. Depending on the balance between the polar and nonpolar parts, a large number of self-assembly structures is

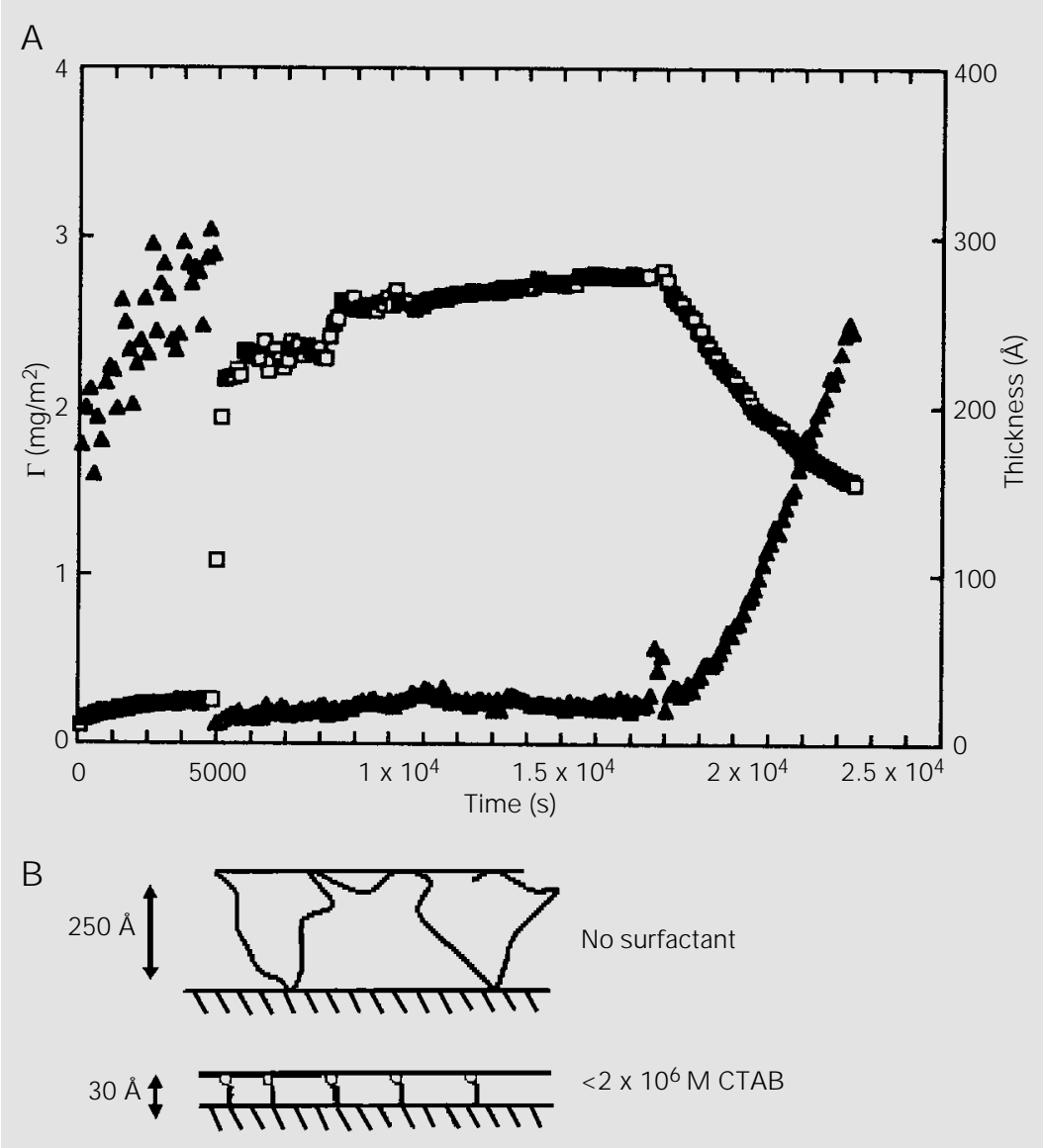

Figure 9. A, Adsorbed amount (open squares) and adsorbed layer thickness (triangles) as a function of time. The DNA (700 bp) is added at time $0(0.06 \mathrm{mg} / \mathrm{ml})$, at $\mathrm{t} 5000 \mathrm{~s}$ cetyltrimethylammonium bromide (CTAB) is added at a concentration of $1.5 \times 10^{-6} \mathrm{M}$. Then the CTAB concentration is increased stepwise up to $t 17,000 \mathrm{~s}$ when rinsing is started. Reprinted from Ref. 40, with permission. B, Schematic representation of the system. With no surfactant added DNA adsorbs slowly onto the surface. With the addition of the surfactant the adsorbed amount increases but the adsorbed layers compact strongly. 

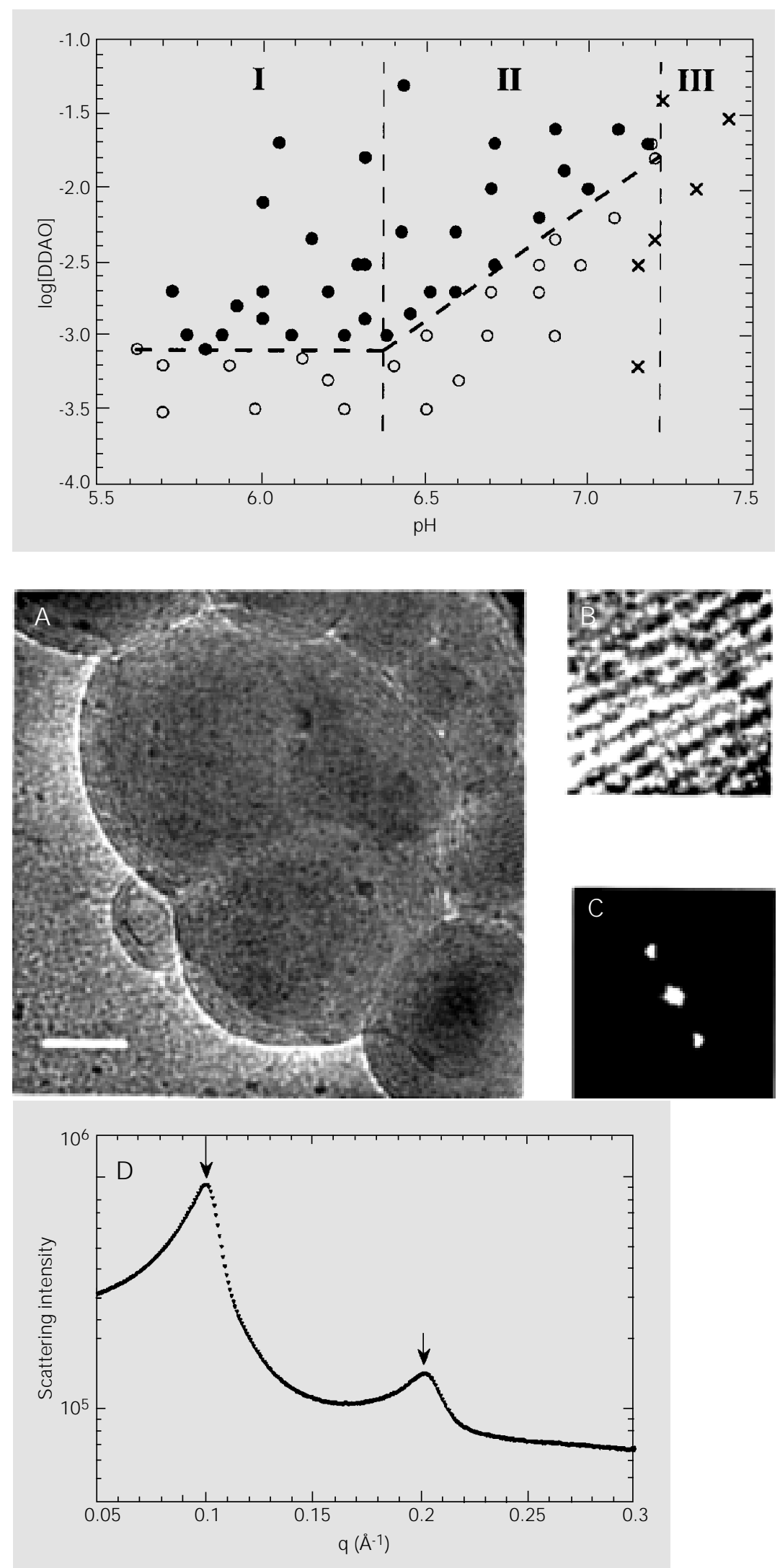

Figure 10. Phase map of the T4 DNA-dodecyldimethylamine oxide (DDAO) system at $25^{\circ} \mathrm{C}$ in $50 \mathrm{mM} \mathrm{NaCl}$ obtained with fluorescence microscopy at various $\mathrm{pH}$ values of the surfactant solution (as measured before mixing with DNA). Coil T4 DNA molecules are represented by crosses, the coexistence of unfolded and condensed T4 DNA molecules is represented by open circles, and the compact globular state of all DNA molecules by closed circles. Reprinted from Ref. 33, with permission.

Figure 11. Multilamellar structures formed in DNAdodecyldimethylamine oxide (DDAO):dioleoylphosphatidylethanolamine (DOPE) mixtures under acidic conditions $(\mathrm{pH}=5.5)$. A, Cryo-TEM image of vitrified DNA-mixed vesicles complexes in $50 \mathrm{mM}$ $\mathrm{NaCl}$ aqueous solutions (scale bar $=90 \mathrm{~nm}$ ). B, Magnified detail of the multilamellar pattern from A. C, Fourier transforms of B. D, Precipitated DNA(DDAO:DOPE) complexes (DNA:DDAO molar ratio is $1: 1)$. Reprinted from Ref. 51 , with permission. 
possible, as established in phase diagram work for many systems (43-45). Only recently have we started to learn about how polymers modify surfactant self-assembly structures $(2,3,9,46)$.

The first studies probing the surfactant aggregates in the concentrated phase in a phase separated polyion-surfactant system revealed by fluorescence that the aggregates are discrete and have low aggregation numbers; they are small globular, closely spherical micelles (47-50). Later work has demonstrated the rich morphology possible. The best studied systems are mixtures of sodium poly(acrylate) with cationic surfactants (8). Here, in addition to spherical micelles, a micellar cubic liquid crystalline phase has been demonstrated as well as hexagonal and lamellar phases. There appear to be no reports for other systems of either bicontinuous cubic phases or reversed structures (reversed hexagonal, cubic or micellar).

For mixed systems of DNA and cationic surfactants, it appears that only lamellar and hexagonal structures have been clearly established by small angle X-ray and neutron diffraction $(3,7)$; the hexagonal phase was inferred to be of the inverse type. Indications in our laboratories of a cubic phase (as inferred by small angle X-ray diffraction) are in line with observations for other polyion-surfactant systems and for simple surfactant systems and are not unexpected, but need to be further studied. Figure 11 illustrates observations of microstructure in DNA-surfactant systems.

The type of surfactant aggregate present is expected to be important, not least for the mechanism of gene transfection using cationic surfactant systems as vectors. In current work the transfection efficiency is generally correlated with the chemical structure of the surfactant/lipid used as vector. In our opinion, the aggregate structure plays a dominant role and correlations of aggregate morphology with transfection efficiency should be more fruitful. Besides the surfactant organization on shorter and longer length scales, we also need to establish how DNA molecules are located, in particular within different periodic surfactant structures.

\section{DNA compaction can be reversed in mixed surfactant systems}

The relevance of investigating DNA compaction in mixed systems of a cationic surfactant and of an anionic surfactant/polymer relates to the control of DNA compaction, to the possibility of reversing compaction, and to the fate of compacted DNA in a biological system, where it will be interacting both with anionic lipids and anionic polysaccharides.

Figure 12 illustrates how a mixed surfac-
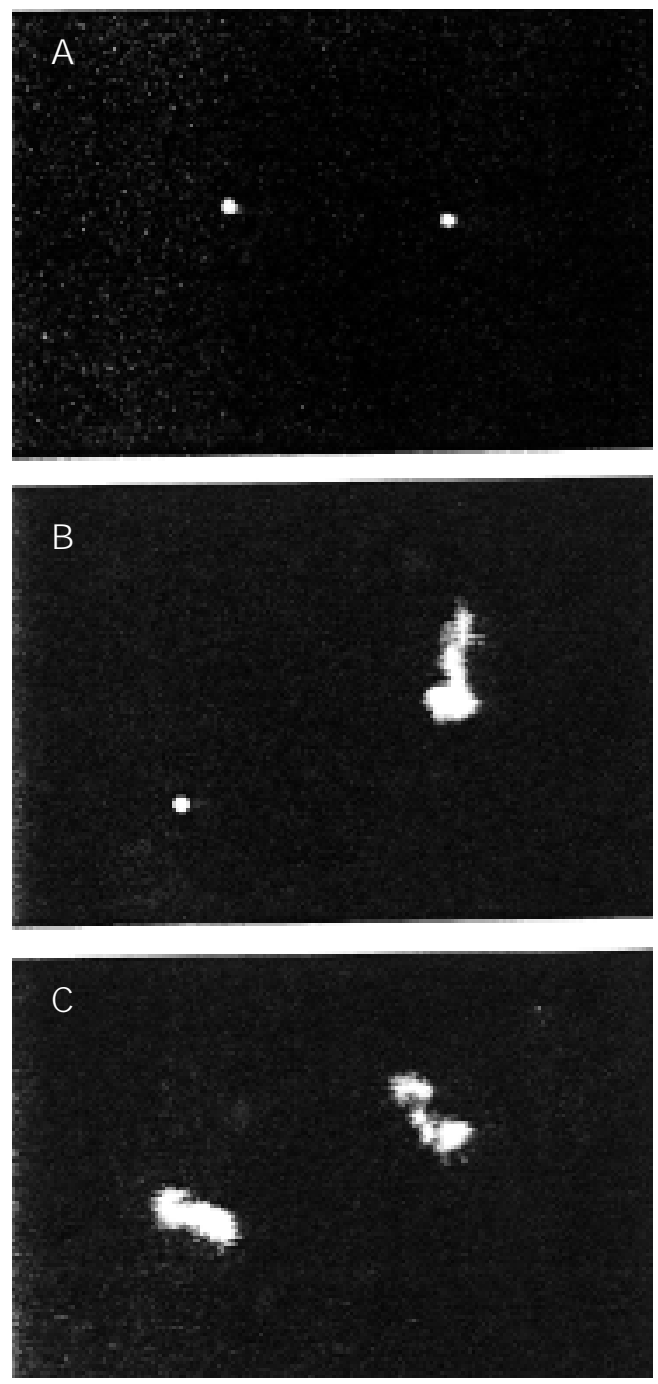

Figure 12. A, T4 DNA molecules in the compacted conformation due to the presence of the cationic surfactant cetyltrimethylammonium bromide (CTAB), in solution. B, For intermediate amounts of the anionic surfactant sodium octylsulfate (SOS), we can see a coexistence between the two DNA conformations, i.e., coils and globules. C, With further addition of SOS all molecules undergo decompaction. $\mathrm{T}=25^{\circ} \mathrm{C}$. 


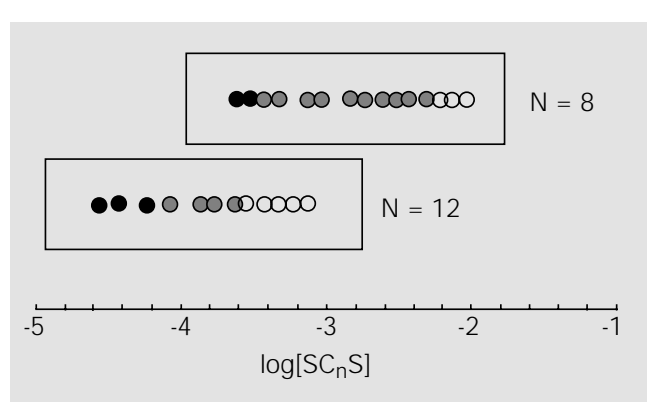

A

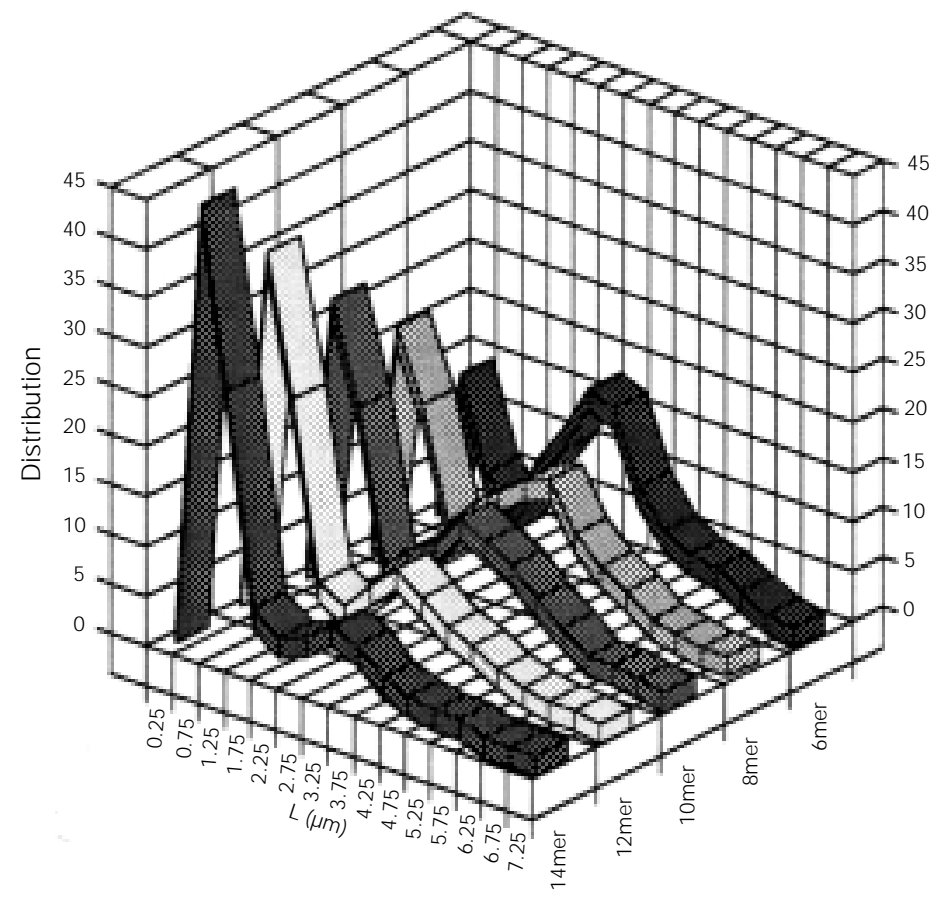

B

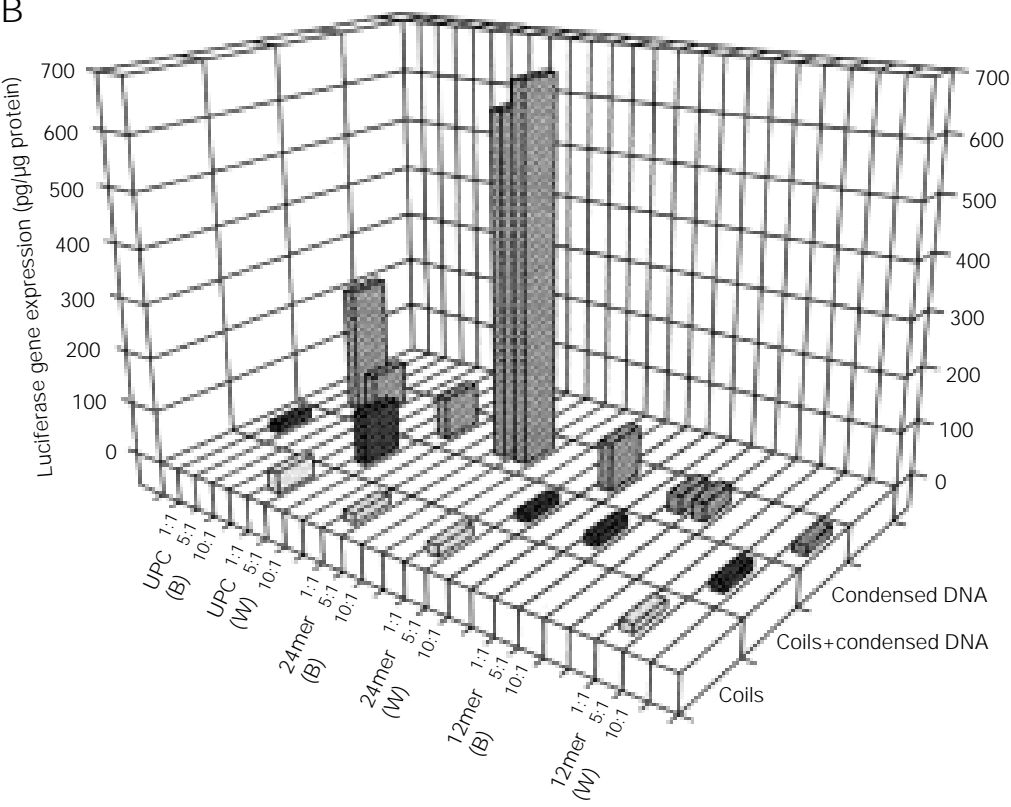

Figure 13. Dependence of the conformational behavior of T4 DNA molecules in aqueous buffer solution at constant $\log [\mathrm{DTAB}]=-3.5$ on the concentration of sodium dodecylsulfate $(\mathrm{N}=12)$ and sodium octylsulfate $(\mathrm{N}=8)$. Filled circles correspond to the globular DNA conformation, shaded circles to the coexistence of elongated coils and compact globules, and open circles to the coiled conformation state of DNA. $T=25^{\circ} \mathrm{C}$. DTAB, dodecyltrimethylammonium bromide.

Figure 14. A, Effect of chitosan chain length (or number of charges) on DNA condensation. A change in distribution of long-axis length of T4 DNA in aqueous solutions of different chitosan oligomers is observed ([chitosan]: [DNA] charge ratio is equal, 5:1). B, Correlation between the transgene expression of chitosan oligomer/ polymer-plasmid DNA complexes and the T4 DNA conformational state for the same oligomer/polymer formulations as visualized by fluorescence microscopy. (W) - Formulations prepared in water (pH 6.1), (B) formulations prepared in acetate buffer ( $\mathrm{pH}$ 5.0). UPC = ultrapure polymeric chitosan. Reprinted from Ref. 51, with permission.

tant system can be used for control of compaction. DNA compacted by means of a cationic surfactant is decompacted when an anionic surfactant is added. Three observations should be noted: first, decompaction occurs as compaction via an intermediate situation of coexistence between coil and globules. Second, even a weakly amphiphilic (in this case an octyl chain) surfactant is effective in decompaction, showing the predominance of surfactant-surfactant interactions over polyion-surfactant interactions; the former involve both hydrophobic and electrostatic interactions. Third, by increasing the hydrophobicity of the negative surfactant, lower amounts of it are required to release DNA into a solution (Figure 13).

The dependence of the positive surfactant chain length on the decompaction, as well as the structures that both positive and negative surfactants form is being studied.

\section{A polycation compacts DNA in a similar way as a surfactant aggregate}

As discussed earlier, the counterion va- 
lency has an important influence on DNA conformation and a standard way of compaction is to use counterions like spermine and spermidine. A polycation will be even more effective and here we will review some results obtained with a cationic polysaccharide, chitosan. In these studies the molecular weight, and thus also the valency, could be varied over wide ranges due to access to well-defined oligomers and polymers (51).

The studies do indeed demonstrate, as exemplified in Figure 14A, the critical role of valency; thus the higher the molecular weight of chitosan, the lower is the concentration needed for compaction. Salt may reverse compaction, but also, as well as lowering of the $\mathrm{pH}$, induce precipitation of DNAchitosan complexes. The studies with chitosan also involved studies of transfection. The general conclusion is that a chitosan effective in promoting compaction is also efficient in transfection (Figure 14B).

\section{Many aspects remain poorly understood}

In reviewing various aspects of our current studies of DNA-surfactant systems we have attempted to identify points where our understanding needs particular advancement. We have seen that basic aspects of the cooperativity in surfactant binding, and important differences with respect to other polyion systems, need further consideration as well as general aspects of phase separation and other features of the phase diagram. The structure of the DNA-surfactant complexes is still a challenge. Also, further studies are being conducted on the compaction/decompaction of DNA, with special emphasis on the use of less toxic lipids.

\section{Acknowledgments}

Collaborations with S. Mel'nikov, Y. Mel'nikova, T. Nylander, C. Leal, and K. Eskilsson are gratefully acknowledged.

\section{References}

1. Lasic DD (1997). Liposomes in Gene Delivery. CRC Press, Boca Raton, FL, USA.

2. Ilekti P, Piculell L \& Cabane B (1998). How to concentrate an aqueous polyelectrolyte/surfactant mixture by adding water. J ournal of Physical Chemistry B, 102: 344351.

3. Ilekti P, Martin T \& Piculell L (1999). Effects of polyelectrolytes on the structures and interactions of surfactant aggregates. J ournal of Physical Chemistry B, 103: 9831-9840.

4. Buckin V, Kudryashow E, Morrissey S, Kapustina T \& Dawson K (1998). Do surfactants form micelles on the surface of DNA? Progress in Colloid and Polymer Sciences, 110: 214-219.

5. Ghirlando R, Wachtel EJ , Arad T \& Minsky A (1992). DNA packaging induced by micellar aggregates: a novel in vitro DNA condensation system. Biochemistry, 31: 7110-7119.

6. Mel'nikov SM, Sergeyev VG \& Yoshikawa K (1997). Visualization of DNA-surfactant interactions with fluorescence microscopy. In: Pandalai SG (Editor), Recent Re- search Developments in Chemical Sciences. Trivandrum, India, 69-113.

7. Rädler J , Koltover I, Salditt T \& Safinya C (1997). Structure of DNA-cationic liposome complexes: DNA intercalation in multilamellar membranes in distinct interhelical packing regimes. Science, 275: 810-814.

8. Svensson A, Piculell L, Cabane B \& llekti $P$ (2002). A new approach to the phase behavior of oppositely charged polymer and surfactant. J ournal of Physical Chemistry $B, 106:$ 1013-1018.

9. Piculell L, Lindman $B \&$ Karlström $G$ (1998). Phase behavior of polymer-surfactant systems. In: Kwak J CT (Editor), Polymer-Surfactant Systems. Marcel Dekker, New York, NY, USA, 65-141.

10. Lindman B \& Thalberg K (1993). Polymersurfactant interactions - recent developments. In: Goddard E \& Ananthapadmanabhan K (Editors), Interactions of Surfactants with Polymers and Proteins. CRC Press, Boca Raton, FL, USA, 203-276.

11. Dias R, Mel'nikov S, Lindman $B \&$ Miguel M (2000). DNA phase behavior in the pres- ence of oppositely charged surfactants. Langmuir, 16: 9577-9583.

12. Malovikova A, Hayakawa K \& Kwak J (1984). Surfactant-polyelectrolyte interactions. 4. Surfactant chain length dependence of the binding of alkylpyridinium cations to dextran sulfate. J ournal of Physical Chemistry, 88: 1930-1933.

13. Thalberg $K$, Lindman $B \&$ Karlström $G$ (1991). Phase behavior of systems of cationic surfactant and anionic polyelectrolyte: influence of surfactant chain length and polyelectrolyte molecular weight. J ournal of Physical Chemistry, 95: 33703376.

14. McLoughlin D (2000). A study of DNAsurfactant complexes in aqueous and aqueous-organic solvents. Doctoral thesis, University College Dublin, Dublin, Ireland.

15. Mel'nikov SM, Sergeyev V, Mel'nikova Y \& Yoshikawa K (1997). Folding of long DNA chains in the presence of distearyldimethylammonium bromide and unfolding induced by neutral liposomes. J ournal of the Chemical Society - Faraday Trans- 
actions, 93: 283-288.

16. Mel'nikov SM, Sergeyev V, Yoshikawa K, Takahashi H \& Hatta I (1997). Cooperativity or phase transition? Unfolding transition of DNA cationic surfactant complex. J ournal of Chemical Physics, 107: 69176924.

17. Efremov VA, Shikina YV \& Khokhlov AR (1992). Disproportionation model for interpolymeric reactions. Polymer Science, 34: 484-486.

18. Goddard E \& Hannan R (1977). Polymer/ surfactant interactions. J ournal of the American Oil Chemists' Society, 54: 561566.

19. Kim B, Ishikawa M, Gong J \& Osada $Y$ (1999). Molecular and supramolecular structures of complexes formed by polyelectrolyte-surfactant interactions - effects of charge density and compositions. J ournal of Polymer Science. A, General Papers, 37: 639-644.

20. Thalberg $K$, Lindman $B \&$ Karlström $G$ (1991). Phase behavior of a system of cationic surfactant and anionic polyelectrolyte: the effect of salt. J ournal of Physical Chemistry, 95: 6004-6011.

21. Marques $E$, Khan $A$, Miguel MG \& Lindman B (1993). Self-assembly of a cationic and an anionic surfactant: the sodium dodecyl sulfate-didodecyldimethylammonium bromide-water system. J ournal of Physical Chemistry, 97: 4729-4736.

22. J önsson $B$, Lindman $B$, Holmberg $K \&$ Kronberg B (1998). Surfactants and Polymers in Aqueous Solution. J ohn Wiley \& Sons, New York, NY, USA.

23. Guldbrand $L$, J önsson $B$, Wennerström $H$ \& Linse P (1984). Electrical double layer forces. A Monte Carlo study. J ournal of Chemical Physics, 80: 2221-2228.

24. Khan MO \& J önsson B (1999). Electrostatic correlations fold DNA. Biopolymers, 49: 121-125.

25. Khan MO, Mel'nikov SM \& J önsson B (1999). Anomalous salt effects on DNA conformation: experiment and theory. Macromolecules, 32: 8836-8840.

26. Khan $A$, J önsson $B \&$ Wennerström $H$ (1985). Phase equilibria in the mixed sodium and calcium di-2-ethylhexylsulfosuccinate aqueous system. An illustration of repulsive and attractive double layer forces. J ournal of Physical Chemistry, 89: 5180-5184.

27. Wennerström $\mathrm{H}$, Khan $\mathrm{A} \&$ \& Lindman $B$ (1991). Ionic surfactants with divalent counterions. Advances in Colloid and Interface Science, 34: 433-449.

28. Yanagida M, Hiraoka Y \& Katsura I (1983). Dynamic behaviors of DNA molecules in solution studied by fluorescence microscopy. Cold Spring Harbor Symposium on Quantitative Biology, 47: 177-187.

29. Bustamante C (1991). Direct observation and manipulation of single DNA molecules using fluorescence microscopy. Annual Review of Biophysics and Biophysical Chemistry, 20: 415-446.

30. Mel'nikov SM, Sergeyev VG \& Yoshikawa K (1995). Discrete coil-globule transition of large DNA induced by cationic surfactant. J ournal of the American Chemical Society, 117: 2401-2408.

31. Mel'nikov SM, Sergeyev VG \& Yoshikawa K (1995). Transition of double-stranded DNA chains between random coil and compact globule states induced by cooperative binding of cationic surfactant. J ournal of the American Chemical Society, 117: 9951-9956.

32. Mel'nikov SM, Sergeyev VG \& Yoshikawa K (1997). Cooperation between salt induced globule-coil transition in single duplex DNA complexed with cationic surfactant and sphere-rod transition of surfactant micelles. Progress in Colloid and Polymer Sciences, 106: 209-214.

33. Mel'nikova YS \& Lindman B (2000). pHcontrolled DNA condensation in the presence of dodecyldimethylamine oxide. Langmuir, 16: 5871-5878.

34. Mel'nikov SM, Khan MO, Lindman B \& J önsson B (1999). Phase behavior of single DNA in mixed solvents. J ournal of the American Chemical Society, 121: 1130-1136

35. Marques EF (2000). Size and stability of catanionic vesicles: effects of formation path, sonication, and aging. Langmuir, 16: 4798-4807.

36. Marques EF, Regev $O$, Khan $A$, Miguel $M$ \& Lindman B (1998). Vesicle formation and general phase behavior in the catanionic mixture SDS-DDAB-water. The anionic-rich side. J ournal of Physical Chemistry, 102: 6746-6758.

37. Marques EF, Regev $O$, Khan $A$, Miguel $M$ \& Lindman B (1999). Vesicle formation and general phase behavior in the catanionic mixture SDS-DDAB-water. The cationic-rich side. J ournal of Physical Chemistry, 103: 8353-8363.

38. Mel'nikov SM, Dias $R$, Mel'nikova $Y$, Marques $M$, Miguel $M \&$ Lindman $B$ (1999). DNA conformational dynamics in the presence of catanionic mixtures. FEBS Letters, 453: 113-118.

39. Yatcilla $M T$, Herrington $K C$, Brasher $L L$, Kaler EW, Chiruvolu S \& Zasadzinski J A (1996). Phase behavior of aqueous mixtures of cetyltrimethylammonium bro- mide (CTAB) and sodium octyl sulfate (SOS). J ournal of Physical Chemistry, 100 : 5874-5879.

40. Eskilsson K, Leal C, Lindman B, Miguel M $\&$ Nylander T (2001). DNA-surfactant complexes at solid surfaces. Langmuir, 17: 1666-1669.

41. Maeda H, Tsunoda M \& Ikeda S (1974). Electric and non-electric interaction of an anionic-cationic micelle. J ournal of Physical Chemistry, 78: 1086-1090.

42. Ikeda S, Tsunoda M \& Maeda H (1978) The application of the Gibbs adsorption isotherm to aqueous solutions of a nonionic-cationic surfactant. J ournal of Colloid and Interface Science, 67: 336-348.

43. Rogers J \& Winsor PA (1969). Change in the optic sign of the lamellar phase $(G)$ in the aerosol OT/water system with composition or temperature. J ournal of Colloid and Interface Science, 30: 247-257.

44. Balmbra RR, Clunie J S \& Goodman J F (1969). Cubic mesomorphic phases. Nature, 222: 1159-1160.

45. Laughlin RG (1994). The Aqueous Phase Behavior of Surfactants. Academic Press, London, UK.

46. Piculell L \& Lindman B (1992). Association and segregation in aqueous polymer/ polymer, polymer/surfactant, and surfactant/surfactant mixtures: similarities and differences. Advances in Colloid and Interface Science, 41: 149-178.

47. Hansson P \& Almgren M (1995). Polyelectrolyte-induced micelle formation of ionic surfactants and binary surfactant mixtures studied by time-resolved fluorescence quenching. J ournal of Physical Chemistry, 99: 16684-16693.

48. Hansson $P$ \& Almgren M (1995). Large C12TAB micelles formed in complexes with polyvinylsulfate and dextran sulfate. J ournal of Physical Chemistry, 99: 1669416703.

49. Hansson P \& Almgren M (1996). Interaction of CnTAB with sodium (carboxymethyl)cellulose: effect of polyion linear charge density on binding isotherms and surfactant aggregation number. J ournal of Physical Chemistry, 100: 9038-9046.

50. Hansson P\& Almgren M (2000). Reliable aggregation numbers are obtained for polyelectrolyte bound cationic micelles using fluorescence quenching with a cationic surfactant quencher. J ournal of Physical Chemistry, 104: 1137-1140.

51. Mel'nikova YS (2000). Association of amphiphiles and polyelectrolytes with DNA. Doctoral thesis, Lund University, Lund, Sweden. 